\title{
Beyond the Standard Model in $B$ Decays: Three Topics ${ }^{1}$
}

\author{
Alexander L. Kagan ${ }^{2}$ \\ Physics Department, University of Cincinnati, Cincinnati OH 45221, USA
}

\begin{abstract}
Three new results are discussed: (a) A non-vanishing amplitude for the 'wrong sign' kaon decay $B \rightarrow J / \Psi \bar{K}$ or its $C P$ conjugate is shown to be a necessary condition for obtaining different CP asymmetries in $B \rightarrow J / \Psi K_{S, L}$. A significant effect would require a scale of new physics far below the weak scale, all but ruling out this possibility. (b) The leading isospin breaking contributions to the $B \rightarrow K^{*} \gamma$ decay amplitudes can be calculated in QCD factorization, providing a sensitive probe of the penguin sector of the effective weak Hamiltonian. New physics models which reverse the predicted $10-20 \%$ Standard Model amplitude hierarchy could be ruled out with more precise data. (c) A slowly falling $g^{*} g \eta^{\prime}$ form factor can be ruled out using the $\eta^{\prime}$ spectrum obtained by ARGUS at the $\Upsilon(1 S)$. The decay $b \rightarrow s g \eta^{\prime}$ is therefore highly suppressed and the origin of the anomalously large $B \rightarrow \eta^{\prime} X_{S}$ rate remains unknown, perhaps requiring the intervention of New Physics.
\end{abstract}

\section{INTRODUCTION}

Three recent developments with potential implications for new physics and $B$ decays are discussed. Recently, the BaBaR and Belle collaborations presented new measurements of the time-dependent CP asymmetries in $B \rightarrow J / \Psi K_{S}$ and $B \rightarrow J / \Psi K_{L}[1,2]$. In the Standard Model the two CP asymmetries are predicted to have the same magnitude but opposite sign. Although the measured values are equal within errors the $\mathrm{CP}$ asymmetry in the $B \rightarrow J / \Psi K_{L}$ channel is somewhat larger, which raises the question of whether $\left|a_{\mathrm{CP}}\left(B \rightarrow \psi K_{L}\right)\right| \neq\left|a_{\mathrm{CP}}\left(B \rightarrow \psi K_{S}\right)\right|$ is possible. The conditions which must be fulfilled for this to happen are explained below [3]. A model-independent analyis implies that the associated scale of new physics interactons would have to lie at a prohibitively low scale of a few $\mathrm{GeV}$ in order to obtain a significant effect.

Measurements of the exclusive $B \rightarrow K^{*} \gamma$ branching ratios have been reported by the CLEO, Belle and BaBar Collaborations, with the results (averaged over CP-conjugate modes):

$$
10^{5} \operatorname{Br}\left(\bar{B}^{0} \rightarrow \bar{K}^{* 0} \gamma\right)= \begin{cases}4.55_{-0.68}^{+0.72} \pm 0.34 & {[4]} \\ 4.96 \pm 0.67 \pm 0.45 & {[5]} \\ 4.23 \pm 0.40 \pm 0.22 & {[6]}\end{cases}
$$

\footnotetext{
${ }^{1}$ Invited talk at the 9th International Symposium on Heavy Flavor Physics, Caltech, Pasadena, Sept. 1013,2001

2 Work supported by the Department of Energy, Grant No. DOE DE FG02-84ER-40153
} 


$$
10^{5} \mathrm{Br}\left(B^{-} \rightarrow \bar{K}^{*-} \gamma\right)= \begin{cases}3.76_{-0.83}^{+0.89} \pm 0.28 & {[4]} \\ 3.89 \pm 0.93 \pm 0.41 & {[5]} \\ 3.83 \pm 0.62 \pm 0.22 & {[6]}\end{cases}
$$

The average branching ratios for the two modes are $(4.44 \pm 0.35) \cdot 10^{-5}$ and $(3.82 \pm$ $0.47) \cdot 10^{-5}$. When corrected for the difference in the $B$-meson lifetimes, $\tau_{B^{-}} / \tau_{\bar{B}^{0}}=$ $1.068 \pm 0.016[7]$, these results imply

$$
\Delta_{0-} \equiv \frac{\Gamma\left(\bar{B}^{0} \rightarrow \bar{K}^{* 0} \gamma\right)-\Gamma\left(B^{-} \rightarrow \bar{K}^{*-} \gamma\right)}{\Gamma\left(\bar{B}^{0} \rightarrow \bar{K}^{* 0} \gamma\right)+\Gamma\left(B^{-} \rightarrow \bar{K}^{*-} \gamma\right)}=0.11 \pm 0.07
$$

Although there is no significant deviation of this quantity from zero, the fact that all three experiments see a tendency for a larger neutral decay rate raises the question of whether the Standard Model can account for isospin-breaking effects of order $10 \%$ in the decay amplitudes.

Recently it has been shown that in the heavy-quark limit the decay amplitudes for these processes can be calculated in a model-independent way using a QCD factorization approach $[8,9]$, which is similar to the scheme developed for the analysis of nonleptonic two-body decays of $B$ mesons [10]. To leading order in $\Lambda / m_{b}$ one finds that the amplitudes for the decays $\bar{B}^{0} \rightarrow \bar{K}^{* 0} \gamma$ and $B^{-} \rightarrow K^{*-} \gamma$ coincide. Here we report on subsequent work [11], in which the QCD factorization approach was used to estimate the leading isospin-breaking contributions for the $B \rightarrow K^{*} \gamma$ decay amplitudes in the Standard Model. These are due to annihilation graphs which enter at order $\Lambda / m_{b}$. Because of their relation to matrix elements of penguin operators, we will see that isospin-breaking effects in $B \rightarrow K^{*} \gamma$ decays are sensitive probes of physics beyond the Standard Model.

Finally, the CLEO collaboration and more recently, as we heard at this workshop, the $\mathrm{BaBaR}$ collaboration have measured very large rates for fast $\eta^{\prime}$ production in $B \rightarrow \eta^{\prime} X_{s}$ decays:

$$
\mathcal{B R}\left(B \rightarrow \eta^{\prime} X_{S}\right)_{p_{\eta^{\prime}}>2 \mathrm{GeV}}= \begin{cases}6.2 \pm 1.6 \pm 1.3_{-1.5}^{+0} \times 10^{-4} ; & \text { CLEO [12] } \\ 6.8_{-1.0}^{+.7} \pm 1_{-.5}^{+0} \times 10^{-4} ; & \text { BaBar [13] }\end{cases}
$$

The experimental cut on $p_{\eta^{\prime}}$ is beyond the kinematic limit for most $b \rightarrow c$ decays. A majority of the events lie at large recoil mass, consistent with a three-body or higher multiplicity decay. The $\eta^{\prime}$ yield from the $b \rightarrow c$ component is dominated by intermediate charmonia decays, which contribute only $\approx 1.1 \times 10^{-4}$ [14] to the branching ratio. Charmless $\eta^{\prime}$ production proceeding via the quark content of the $\eta^{\prime}$ has been estimated using factorization $[15,16]$, giving a contribution to the branching ratio of $\approx 1 \times 10^{-4}$ with a quasi two-body recoil spectrum that is peaked at low energies in conflict with the observed spectrum.

The surprisingly large $\eta^{\prime}$ yield led Atwood and Soni [14] to propose that it is associated with the gluonic content of the $\eta^{\prime}$ via the subprocess $b \rightarrow s\left(g^{*} \rightarrow \eta^{\prime} g\right)$. Making the key assumption that the form factor remains constant up to $q^{2} \sim m_{b}^{2}$, where $q$ is the virtual gluon's momentum, they showed that the large $\eta^{\prime}$ yield could easily be reproduced in the Standard Model. Moreover, they observed that the three-body decay leads to an $\eta^{\prime}$ recoil spectrum that is consistent with observation. Hou and Tseng [17] argued that the 
factor of $\alpha_{s}$ implicit in $H$ should be evaluated at the scale of momentum transfer through the $g^{*} g \eta^{\prime}$ vertex. However this would only introduce a mild logarithmic suppression of the form factor versus $q^{2}$ and therefore still lead to a large $\eta^{\prime}$ yield.

Assymptotically, perturbative QCD (pQCD) predicts that the leading form factor contributions should fall like $1 / q^{2}$. The question is in what region of $q^{2}$ does this behaviour set in? We will see [20] that the $\eta^{\prime}$ spectrum measured in $\Upsilon(1 S) \rightarrow \eta^{\prime} X$ decays by the ARGUS Collaboration [18] rules out form factors which fall slowly in the range $q^{2} \leq m_{b}^{2}$. However, a rapidly falling form factor [16] representative of pQCD predictions $[21,22]$ is consistent with the data. The corresponding $b \rightarrow s g \eta^{\prime}$ branching ratio in the Standard Model is about a factor of 20 smaller than the measured values in Eq. (2).

\section{CAN THE CP ASYMMETRIES IN $B \rightarrow J / \Psi K_{S, L}$ BE DIFFERENT?}

The relevant quantities are [23]

$$
\lambda_{S, L} \equiv \frac{q_{B}}{p_{B}} \frac{\bar{A}_{S, L}}{A_{S, L}}
$$

where

$$
\bar{A}_{S, L} \equiv A\left(\bar{B} \rightarrow \psi K_{S, L}\right), \quad A_{S, L} \equiv A\left(B \rightarrow \psi K_{S, L}\right) .
$$

The neutral $\mathrm{B}$ and $\mathrm{K}$ meson mass eigenstates are defined in the usual way in terms of flavor eigenstates,

$$
\left|B_{L, H}\right\rangle=p_{B}|B\rangle \pm q_{B}|\bar{B}\rangle, \quad\left|K_{S, L}\right\rangle=p_{K}|K\rangle \pm q_{K}|\bar{K}\rangle .
$$

The time-dependent $\mathrm{CP}$ asymmetries are given by

$$
a_{\mathrm{CP}}\left(B \rightarrow \psi K_{S, L}\right)=-2 \frac{\operatorname{Im} \lambda_{S, L}}{1+\left|\lambda_{S, L}\right|^{2}} \sin \Delta m_{B} t+\frac{1-\left|\lambda_{S, L}\right|^{2}}{1+\left|\lambda_{S, L}\right|^{2}} \cos \Delta m_{B} t .
$$

In the limit of no direct $\mathrm{CP}$ violation $\left(\left|\lambda_{S, L}\right|=1\right)$ the asymmetries reduce to the simple form $a_{\mathrm{CP}}\left(B \rightarrow \psi K_{S, L}\right)=-\operatorname{Im} \lambda_{S, L} \sin \Delta m_{B} t$, as in the Standard Model.

We need to rewrite $\lambda_{S, L}$ in terms of the decay amplitudes into kaon flavor eigenstates,

$$
\begin{aligned}
& \bar{A}_{K} \equiv A(\bar{B} \rightarrow \psi K), \quad \bar{A}_{\bar{K}} \equiv A(\bar{B} \rightarrow \psi \bar{K}), \\
& A_{K} \equiv A(B \rightarrow \psi K), \quad A_{\bar{K}} \equiv A(B \rightarrow \psi \bar{K}) \text {. }
\end{aligned}
$$

Allowing for the possibility that the 'wrong-sign' kaon amplitudes $\bar{A}_{K}$ and $A_{\bar{K}}$ receive new physics contributions (they are negligible in the Standard Model), one obtains

$$
\lambda_{S, L}= \pm \lambda\left(\frac{1 \pm a}{1 \pm b}\right)
$$


where $\lambda \equiv q_{B} q_{K} \bar{A}_{\bar{K}} / p_{B} p_{K} A_{K}$, and $a$ and $b$ are proportional to ratios of wrong-sign to right-sign kaon amplitudes

$$
a \equiv \frac{p_{K} \bar{A}_{K}}{q_{K} \bar{A}_{\bar{K}}}, \quad b \equiv \frac{q_{K} A_{\bar{K}}}{p_{K} A_{K}} .
$$

In the Standard Model and more generally, in any model in which the wrong-sign amplitudes are negligibly small $(a=b=0)$, this reduces to $\lambda_{S, L}= \pm \lambda$ so that $\operatorname{Im}\left(\lambda_{S}+\right.$ $\left.\lambda_{L}\right)=0$. The $\sin \Delta m_{b} t$ term therefore has equal magnitude but opposite sign for the two $\mathrm{CP}$ asymmetries.

From the general relation $\lambda_{S}+\lambda_{L}=\lambda 2(a-b) /\left(1-b^{2}\right)$ we learn that a necessary and sufficient condition for $\lambda_{S} \neq \lambda_{L}$ to be satisfied is the presence of non-vanishing wrong-sign amplitudes with $a \neq b$. As an example, if each of the right-sign and wrongsign amplitudes is dominated by a single contribution, then $|a| \approx|b|$. If in addition, $\operatorname{Re} \lambda \sim \operatorname{Im} \lambda \sim O(1)$, as in the Standard Model, and $\operatorname{Arg}[a] \sim \operatorname{Arg}[b] \sim O(1)$, we obtain $\operatorname{Im}\left(\lambda_{S}+\lambda_{L}\right) \sim|a| \sim|b|$. Thus the difference in CP asymmetries is of the order of the ratio of wrong-sign to right-sign kaon amplitudes.

\section{Constraints on New Physics Scenarios}

Consider the wrong-sign decay $B^{0} \rightarrow J / \Psi \bar{K}$. As the final state does not contain a $d$ quark, the decay must proceed via annihilation of the $B$ meson. It must therefore be mediated by six-quark operators, with an effective Hamiltonian of the form

$$
\frac{g}{M^{5}} \operatorname{ssc} \bar{c} \bar{d} \bar{d}
$$

where $g$ is a dimensionless coupling, $M$ is the scale of new physics ( the color indices and Dirac structure of the operators have been suppressed). An estimate of the wrong-sign amplitude in the factorization approximation yields

$$
A_{\bar{K}} \sim \frac{g}{M^{5}} f_{B} f_{K} f_{\Psi} m_{B} m \Psi\left(\varepsilon \Psi \cdot p_{K}\right) .
$$

For purposes of comparison, we note that in the Standard Model the right-sign amplitude in the factorization approximation is given by

$$
A_{K}=\frac{G_{F}}{\sqrt{2}} V_{c b} a_{2} f_{\Psi} F_{1} m_{\Psi}\left(\varepsilon_{\Psi} \cdot p_{K}\right),
$$

where $F_{1}$ is the $B \rightarrow K$ form factor, and $a_{2}$ is a function of the current-current operator Wilson coefficients $C_{1}$ and $C_{2}$ arising from $W$ exchange. The observed $B \rightarrow J / \Psi K_{S}$ amplitude is reproduced if $a_{2} \approx .25$.

We can get an order of magnitude upper bound on the quantity $g / M^{2}$ by integrating out the charm quarks at one-loop, yielding a contribution to the effective Hamiltonian mediating charmless hadronic $B$ decays of order

$$
\frac{1}{16 \pi^{2}} \frac{g}{M^{2}} b s \bar{d} \bar{d}
$$


Upper bounds on the strenghths of such operators can be obtained by considering their contributions to the rare decays $B^{ \pm} \rightarrow \pi^{ \pm} K_{S}$ or, more specifically, to the wrong-sign kaon decays $B^{ \pm} \rightarrow \pi^{ \pm} \bar{K}^{0}$ [24] in the factorization approximation, yielding $g / M^{2} \lesssim$ $10^{-5} \mathrm{GeV}^{-2}$. Inserting this bound into our estimate for $A_{\bar{K}}$ in Eq. (12) and equating the result with the expression for $A_{K}$ in Eq. (13) (for $a_{2} \approx .25$ ) implies that a scale of new physics $M$ of a few $\mathrm{GeV}$ is required in order to obtain significant wrong-sign kaon amplitudes.

We know of only one scenario with such a potentially low scale for new flavorchanging interactions: supersymmetric models with a light bottom squark $\tilde{b}$ of mass 2-5.5 GeV and light gluinos of mass $12-16 \mathrm{GeV}$ [25], which have been proposed to enhance the $b$ quark production cross section at hadron colliders. Among the new operators which can arise at tree-level are several of the form $\bar{d} b \tilde{b}^{*} \tilde{b}$. Stringent upper bounds on their strenghths from rare $B$ decays have been obtained in [26]. Interactions of the desired form in Eq. (11) would be generated from these operators if the $R$-parity violating Yukawa couplings mediating $\tilde{b} \rightarrow \bar{c} \bar{d}$ and $\tilde{b} \rightarrow \bar{c} \bar{s}$ decays were also present. Unfortunately, an upper bound of order $10^{-5}$ on the product of these two couplings from box-graph contributions to $K-\bar{K}$ mixing implies that the wrong-sign kaon amplitudes would be negligibly small, i.e., $a \sim b \sim 10^{-5}$. Although this result does not constitute a no-go theorem, it appears that the possibility of significantly different $\mathrm{CP}$ asymmetries in $B \rightarrow J / \Psi K_{S, L}$ decays is extremely unlikely due to the requirement of such a low scale for new interactions.

\section{ISOSPIN VIOLATION IN $B \rightarrow K^{*} \gamma$}

In the Standard Model, the effective weak Hamiltonian for $b \rightarrow s \gamma$ transitions is

$$
\mathcal{H}_{\mathrm{eff}}=\frac{G_{F}}{\sqrt{2}} \sum_{p=u, c} \lambda_{p}^{(s)}\left(C_{1} Q_{1}^{p}+C_{2} Q_{2}^{p}+\sum_{i=3, \ldots, 8} C_{i} Q_{i}\right),
$$

where $\lambda_{p}^{(s)}=V_{p s}^{*} V_{p b}, Q_{1,2}^{p}$ are the current-current operators, $Q_{3, \ldots, 6}$ are the local fourquark QCD penguin operators, and $Q_{7}$ and $Q_{8}$ are the electro-magnetic and chromomagnetic dipole operators. (We adopt the conventions of [10]; in particular, $C_{1} \approx 1$ is the largest coefficient.) The Wilson coefficients $C_{i}$ and the matrix elements of the renormalized operators $Q_{i}$ depend on the renormalization scale $\mu$.

At leading power in $\Lambda / m_{b}$ the $B \rightarrow K^{*} \gamma$ decay amplitude is given by

$$
i \mathcal{A}_{\text {lead }}=\frac{G_{F}}{\sqrt{2}} \lambda_{c}^{(s)} a_{7}^{c}\left\langle\bar{K}^{*}(k, \eta) \gamma(q, \varepsilon)\left|Q_{7}\right| \bar{B}\right\rangle,
$$

where the next-to-leading order (NLO) result for the coefficient $a_{7}^{c}=C_{7}+\ldots$ can be found in [8]. The ellipses denote $O\left(\alpha_{s}\right)$ hard spectator interaction contributions.

The leading isospin-breaking effects arise from the diagrams shown in Fig. 1. Their contributions to the decay amplitudes can be parametrized as $\mathcal{A}_{q}=b_{q} \mathcal{A}_{\text {lead }}$, where $q$ is the flavor of the spectator antiquark in the $\bar{B}$ meson. We neglect NLO terms of order $\alpha_{s} C_{3, \ldots, 6}$ while retaining terms of order $\alpha_{s} C_{1,8}$. This is justified, because the penguin 

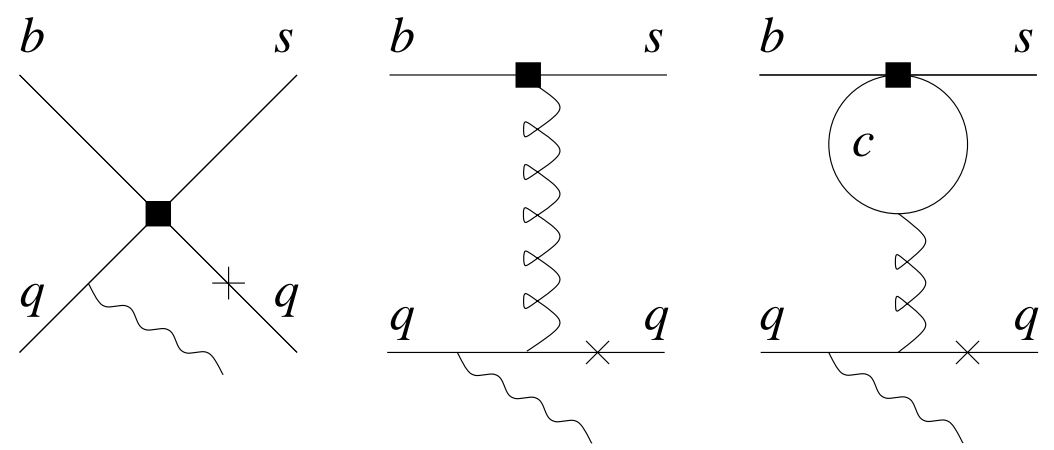

FIGURE 1. Spectator-dependent contributions from local 4-quark operators (left), the chromomagnetic dipole operator (center), and the charm penguin (right). Crosses denote alternative photon attachments.

coefficients $C_{3, \ldots, 6}$ are numerically very small. Also, it is a safe approximation to neglect terms of order $\alpha_{s} \lambda_{u}^{(s)} / \lambda_{c}^{(s)}$. It then suffices to evaluate the contributions of the 4-quark operators shown in the first diagram at tree level.

The QCD factorization approach gives an expression for the coefficients $b_{q}$ in terms of convolutions of hard-scattering kernels with light-cone distribution amplitudes for the $K^{*}$ and $B$ mesons. The result can be written as

$$
b_{q}=\frac{12 \pi^{2} f_{B} Q_{q}}{m_{b} T_{1}^{B \rightarrow K^{*}} a_{7}^{c}}\left(\frac{f_{K^{*}}^{\perp}}{m_{b}} K_{1}+\frac{f_{K^{*} m_{K^{*}}}}{6 \lambda_{B} m_{B}} K_{2}\right),
$$

where $T_{1}^{B \rightarrow K^{*}}$ is a form factor in the decomposition of the $B \rightarrow K^{*}$ matrix element of the tensor current, and $m_{b}$ denotes the running $b$-quark mass. $\lambda_{B}$ is a hadronic parameter which enters the first inverse moment of the relevant $B$ meson distribution amplitude. Heavy-quark scaling laws imply that $b_{q}$ scales like $\Lambda / m_{b}$. However, because of the large numerical factor $12 \pi^{2}$ in the numerator the values of $b_{q}$ turn out to be larger than anticipated in $[8,9]$.

The dimensionless quantities $K_{1}$ and $K_{2}$ are linear functions of the Wilson coefficients. The latter are multiplied by convolution integrals of hard scattering kernels with meson distribution amplitudes. The integrals associated with the four-quark QCD penguin operators and current-current operators exist for any reasonable choice of the distribution amplitudes, which shows that the QCD factorization approach holds at subleading power for their matrix elements, to the order we are working. However, the convolution integral associated with the chromomagnetic dipole operator suffers from a logarithmic end point singularity, indicating that at subleading power factorization breaks down for this matrix element. We regulate the singularity by introducing a cutoff. A large uncertainty is assigned to this estimate since this contribution must be dominated by soft physics.

To leading-order in small quantities the theoretical expression for the isospin-breaking parameter (see Eq. (1)) is $\Delta_{0-}=\operatorname{Re}\left(b_{d}-b_{u}\right)$. A dominant uncertainty in the prediction for $\Delta_{0}$ comes from the tensor form factor $T_{1}^{B \rightarrow K^{*}}$, recent estimates of which range from $0.32_{-0.02}^{+0.04}$ [27] to $0.38 \pm 0.06$ [28]. On the other hand, a fit to the $B \rightarrow K^{*} \gamma$ branching fractions yields the lower value $0.27 \pm 0.04$ [9]. To good approximation the result for 
$\Delta_{0-}$ is inversely proportional to the value of the form factor. We take $T_{1}^{B \rightarrow K^{*}}=0.3$ (at $\mu=m_{b}$ ) as a reference value. Values for the remaining input parameters together with their uncertainties can be found in Ref. [11].

Combining all sources of uncertainty we obtain the Standard Model result

$$
\Delta_{0-}=\left(8.0_{-3.2}^{+2.1}\right) \% \times \frac{0.3}{T_{1}^{B \rightarrow K^{*}}} .
$$

The three largest contributions to the error from input parameter variations are due to $\lambda_{B}\left({ }_{-2.5}^{+1.0} \%\right)$, the divergent integral in the matrix element of $Q_{8}( \pm 1.2 \%)$, and the decay constant $f_{B}( \pm 0.8 \%)$. The perturbative uncertainty is about $\pm 1 \%$. Our result is in good agreement with the current central experimental value of $\Delta_{0-}$ including its sign, which is predicted unambiguously. By far the most important source of isospin-breaking is due to the four-quark penguin operator $Q_{6}$, whose contribution to $\Delta_{0-}$ is about $9 \%$ (at $\mu \approx m_{b}$ ). The other terms are much smaller. In particular, the contribution of the chromo-magnetic dipole operator, for which factorization does not hold, is less than $1 \%$ in magnitude and therefore numerically insignificant. Hence, the important isospin-breaking contributions can be reliably calculated using QCD factorization. It follows from our result that these effects mainly test the magnitude and sign of the ratio $\operatorname{Re}\left(C_{6} / C_{7}\right)$ of penguin coefficients.

Because of their relation to matrix elements of penguin operators, isospin-breaking effects in $B \rightarrow K^{*} \gamma$ decays are sensitive probes of physics beyond the Standard Model. In particular, scenarios in which the sign of $\Delta_{0-}$ is flipped could be ruled out in the near future with more precise data. For simplicity lets restrict ourselves to new physics models which do not enlarge the Standard Model operator basis, where this possibility corresponds to flipping the sign of $\operatorname{Re}\left(C_{6} / C_{7}\right)$. As a specific example, consider the minimal supersymmetric standard model (MSSM) with minimal flavor violation, and with $\tan \beta$ enhanced contributions to $B \rightarrow X_{s} \gamma$ decays taken into account beyond leadingorder [29]. In this scenario new contributions to $Q_{3, \ldots, 6}$, and $Q_{8}$ are too small to have a significant effect. For low $\tan \beta, \operatorname{Re} C_{7}\left(m_{b}\right)$ is negative as in the Standard Model. However, for $\tan \beta \gtrsim 27 \operatorname{Re}_{7}\left(m_{b}\right)$ can take on both positive or negative values with positive values becoming more probable as $\tan \beta$ increases [30]. Therefore, isospin breaking could rule out significant regions of MSSM parameter space at large $\tan \beta$. The sign of $\operatorname{Re}_{7}$ can also be flipped in supersymmetric models with non-minimal flavor violation (independently of $\tan \beta$ ) via gluino/down-type squark loop graphs, without affecting the sign of $C_{6}$. A more detailed treatment of new physics effects, including the possibility of an enlarged operator basis will be presented elsewhere.

\section{CONSTRAINTS ON THE $G^{*} G \eta^{\prime}$ FORM FACTOR FROM $\Upsilon(1 S)$ DECAYS}

The effective $\eta^{\prime} g^{*} g$ coupling can be written as

$$
H\left(q^{2}\right) \varepsilon_{\alpha \beta \mu v} q^{\alpha} k^{\beta} \varepsilon_{1}^{\mu} \varepsilon_{2}^{v},
$$

where $q=p_{b}-p_{s}$ is the virtual gluon's momentum, $k$ is the 'on-shell' gluon's momentum, and $H\left(q^{2}\right)$ is the $g^{*} g \eta^{\prime}$ transition form factor. The QCD axial anomaly determines 


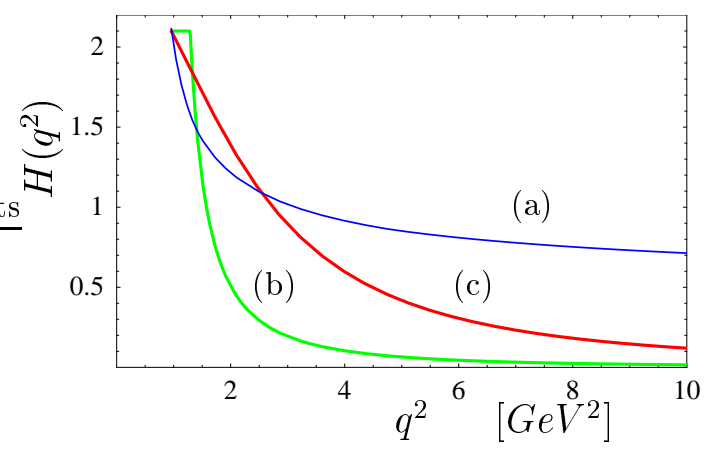

FIGURE 2. Three choices for the form factor $H\left(q^{2}\right)$, as described in the text: (a) the slowly falling form factor, (b) a rapidly falling form factor representative of perturbative QCD calculations, (c) an intermediate example.

the form factor at small momentum transfer, i.e., in the $q^{2} \rightarrow 0$ limit. An estimate of this limit from the decay rate for $J / \psi \rightarrow \eta^{\prime} \gamma$ gives [14] $H(0) \approx 1.8 \mathrm{GeV}^{-1}$.

As we have discussed, the crucial issue that needs to be addressed in determining the $b \rightarrow s g \eta^{\prime}$ decay rate is the dependence of $H$ on $q^{2}$. A simple model for the $g g \eta^{\prime}$ vertex [16] in which a pseudoscalar current is coupled perturbatively to two gluons through quark loops yields a form factor which can be parametrized as

$$
H\left(q^{2}\right)=\frac{H_{0} m_{\eta^{\prime}}^{2}}{q^{2}-m_{\eta^{\prime}}^{2}} .
$$

The dependence of $H_{0}$ on $q^{2}$ is subleading, but it insures the absence of a pole at $q^{2}=m_{\eta^{\prime}}^{2}$. To first approximation it can be modeled by a constant to be identified with the low energy coupling extracted from $J / \psi \rightarrow \eta^{\prime} \gamma$, e.g., $H_{0} \approx 1.8 \mathrm{GeV}^{-1}$. The above parametrization agrees well with recent $\mathrm{pQCD}$ calculations of the form factor $[21,22]$ in which hard amplitudes involving quark and gluon exchanges are convoluted with the $\eta^{\prime}$ quark and gluon wave functions, particularly if $H_{0} \approx 1.7 \mathrm{GeV}^{-1}$.

Below we will consider three representative choices for $H\left(q^{2}\right)$ :

a) The slowly falling form factor of Ref. [17], $H\left(q^{2}\right)=\sqrt{N_{f}} \alpha_{s}\left(q^{2}\right) \cos \theta /\left(\pi f_{\eta^{\prime}}\right) \approx$ $2.1 \alpha_{s}\left(q^{2}\right) / \alpha_{s}\left(m_{\eta^{\prime}}^{2}\right) \mathrm{GeV}^{-1}$ ( $\theta$ is the pseudoscalar mixing angle). It gives $\operatorname{BR}(b \rightarrow$ $\left.s g \eta^{\prime}\right) \approx 6.8 \times 10^{-4}$ for $p_{\eta^{\prime}}>2 \mathrm{GeV}$.

b) The rapidly falling form factor of Eq. (20), with $H_{0} \approx 1.7 \mathrm{GeV}^{-1}$ (at low $q^{2}$ it is matched onto the value of the previous form factor at $\left.q^{2}=m_{\eta^{\prime}}^{2}\right)$. It gives $\operatorname{BR}(b \rightarrow$ $\left.s g \eta^{\prime}\right) \approx 3 \times 10^{-5}$ for $p_{\eta^{\prime}}>2 \mathrm{GeV}$.

c) An intermediate purely phenomenological form factor $H\left(q^{2}\right) \propto 1 /\left(q^{2}+M^{2}\right)$ with $M=2.2 \mathrm{GeV}$, which gives $\mathrm{BR}\left(b \rightarrow s g \eta^{\prime}\right) \approx 4.4 \times 10^{-4}$ for $p_{\eta^{\prime}}>2 \mathrm{GeV}$.

The three form factors are plotted in Fig. 2.

The $g^{*} g \eta^{\prime}$ form factor induces the decay ${ }^{3} S_{1}^{[1]} \rightarrow g g\left(g^{*} \rightarrow \eta^{\prime} g\right)$ of the dominant colorsinglet $\Upsilon(1 S)$ Fock state. Moreover, the region of $q^{2}$ relevant for fast $\eta^{\prime}$ production in this 
decay has large overlap with the region of $q^{2}$ relevant for fast $\eta^{\prime}$ production in $b \rightarrow s g \eta^{\prime}$ decays. Therefore, the $\eta^{\prime}$ spectrum in $\Upsilon(1 S)$ decays could potentially constrain the $g^{*} g \eta^{\prime}$ form factor, and at the same time tell us if the subprocess $b \rightarrow s g \eta^{\prime}$ can account for the $\eta^{\prime}$ yield in $B$ decays [20].

The ARGUS collaboration mesured inclusive $\eta^{\prime}$ production at the $\Upsilon(1 S)$ [18]. Unfortunately, due to limited it was not possible to subtract the continuum contribution from the $\eta^{\prime}$ spectrum, so that modeling of the direct $\Upsilon(1 S)$ decay and continuum components was required. The term 'direct' refers to hadronic $\Upsilon(1 S)$ decays with $e^{+} e^{-} \rightarrow \Upsilon(1 S) \rightarrow q \bar{q}$ events subtracted (the latter are included in the continuum data sample), essentially leaving $\Upsilon(1 S) \rightarrow g g g$ events. The ARGUS fit for direct decays gives $d n / d z \approx 16.8 \beta e^{-10.6 z}$, where $n$ is the $\eta^{\prime}$ multiplicity, and $z \equiv 2 E_{\eta^{\prime}} / m_{\Upsilon}$. However, it is possible to obtain a model-independent upper bound on the $\eta^{\prime}$ multiplicity in each $z$ bin by assuming that the raw $\eta^{\prime}$ yield is entirely due to direct $\Upsilon(1 S)$ decays (note that the total number of direct $\Upsilon(1 S)$ decays in the data sample is known). ${ }^{3}$ Of particular interest is the bound for the highest energy bin $(.7<z<1.0)$ for which we obtain $n_{z>.7}<(6.5 \pm 1.3) \times 10^{-4}$, where the error is statistical only. The model-dependent fit gives a slightly larger result, $8.8 \times 10^{-4}$. We note here that in the near future the CLEO and CLEO-C collaborations will be able to measure the $\eta^{\prime}$ spectrum in $\Upsilon(1 S)$ decays far more precisely.

We have calculated the $\eta^{\prime}$ spectrum, $d \Gamma\left({ }^{3} S_{1}^{[1]} \rightarrow g g g \eta^{\prime}\right) / d z$ for each of the three $g^{*} g \eta^{\prime}$ form factors considered above at leading-order in QCD, using a four-body phase space Monte Carlo [20]. Relativistic corrections have not been taken into account. The spectra have been normalized with respect to the leading-order three gluon decay width, $\Gamma\left({ }^{3} S_{1}^{[1]} \rightarrow g g g\right)$, to obtain the leading-order $\eta^{\prime}$ multiplicities. The results are compared to the ARGUS fit in Fig. 3. Furthermore, comparison with the model-independent ARGUS upper bound for $z>.7$ gives

$$
\left(\frac{n^{\text {theory }}}{n^{\text {argus }}}\right)_{z>.7} \gtrsim \begin{cases}41 & \text { (a) slowly falling, } \\ 1 & \text { (b) pQCD, } \\ 13 & \text { (c) intermediate. }\end{cases}
$$

Evidently, only the rapidly falling (pQCD) form factor is consistent with the ARGUS data at large energies. The intermediate and slowly falling form factors are in gross conflict, giving order of magnitude or greater excesses. Note that the bulk of the $\eta^{\prime}$ yield is expected to originate from long-distance fragmentation of the three gluon configuration and should therefore be quite soft, in agreement with the ARGUS data.

We have checked that significant contributions from decays of higher Fock states of the $\Upsilon(1 S)$ induced by the $g^{*} g \eta^{\prime}$ coupling, e.g., ${ }^{1} S_{0}^{[8]},{ }^{3} P_{J}^{[8]} \rightarrow g g \eta^{\prime}$, would only harden the $\eta^{\prime}$ spectra. Furthermore, relativistic corrections, next-to-leading order QCD corrections, and other theoretical refinements would be insufficient to eliminate the large excesses for $z>.7$ in Eq. (21). Finally, we find that even in the extreme case that the gluon is given a 'phenomenological' $1 \mathrm{GeV}$ mass, as advocated in Ref. [31] (to improve agreement

\footnotetext{
${ }^{3}$ I am grateful to Axel Lindner and Dietrich Wegener for supplying the raw $\eta^{\prime}$ yields and efficiencies from Ref. [19].
} 


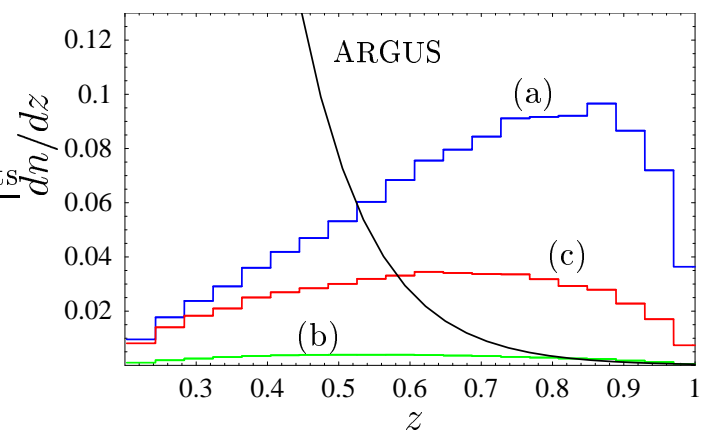

FIGURE 3. The $\eta^{\prime}$ multiplicity spectrum $d n / d z$ in direct $\Upsilon(1 S)$ decays for the three $g^{*} g \eta^{\prime}$ form factors (a) Slowly falling, (b) pQCD (c) intermediate, and the model-dependent ARGUS fit, as described in the text.

between the predicted and observed photon spectra near the end-point for $\Upsilon(1 S)$ decays), the slowly falling form factor still gives an order of magnitude excess.

\section{CONCLUSION}

We have seen that a necessary condition for obtaining significantly different timedependent $\mathrm{CP}$ asymmetries in $B \rightarrow J / \Psi K_{S, L}$ is a non-vanishing amplitude for the 'wrong sign' kaon decay $B \rightarrow J / \Psi \bar{K}$ or its $\mathrm{CP}$ conjugate. We note that it may be possible to test directly for the presence of these amplitudes by searching for wrong-sign $B^{0} \rightarrow J / \Psi\left(\bar{K}^{*} \rightarrow K^{+} \pi^{-}\right)$decays. A model-independent analysis shows that the scale of new physics needs to be prohibitively low, of order a few $\mathrm{GeV}$, all but ruling out this possibility. The only potential example we have found, in the framework of supersymmetric models with an ultra-light bottom squark and light gluinos, would lead to gross violation of $K-\bar{K}$ mixing constraints.

We have seen that isospin breaking effects in $B \rightarrow K^{*} \gamma$ decays can be calculated model-indepndently in the QCD factorization framework. In the Standard Model the decay rate for $\bar{B}^{0} \rightarrow \bar{K}^{* 0} \gamma$ is predicted to be about $10-20 \%$ larger than that for $B^{-} \rightarrow$ $K^{*-} \gamma$, in agreement with the measured central values. The direction of the inequality between these amplitudes is particularly sensitive to the sign of the Wilson coefficient ratio $\operatorname{Re}\left(C_{6} / C_{7}\right)$. As an application, more precise data which will be available in the not too-distant future could rule out models in which the sign of $\operatorname{Re} C_{7}$ is flipped relative to the Standard Model.

Finally, we have seen that slowly falling $g^{*} g \eta^{\prime}$ form factors which can explain the large $B \rightarrow \eta^{\prime} X_{S}$ rate via the subprocess $b \rightarrow s\left(g^{*} \rightarrow g \eta^{\prime}\right)$ are ruled out by ARGUS data on fast $\eta^{\prime}$ production in $\Upsilon(1 S)$ decays. However, the rapidly falling form factor predicted by perturbative QCD is compatible. Unfortunately, the corresponding $B \rightarrow \eta^{\prime} X_{s}$ rate for $p_{\eta^{\prime}}>2 \mathrm{GeV}$ is a factor of 20 smaller than observed. An enhanced $b \rightarrow s g$ chromomagnetic dipole operator, motivated by the low semileptonic branching ratio and charm multiplicity in $B$ decays, could improve agreement with experiment [16]. The CLEO and CLEO-C collaborations will be able to make much more precise measurements of the $\eta^{\prime}$ 
spectrum in $\Upsilon(1 S)$ decays in the near future, potentially providing even more stringent constraints on the $g^{*} g \eta^{\prime}$ form factor.

\section{ACKNOWLEDGMENTS}

It is a pleasure to thank my collaborators on the works presented here, Yixiong Chen, Yuval Grossman, Matthias Neubert, and Zoltan Ligeti. I am grateful to the organizers for arranging a very stimulating symposium, under very trying circumstances. I would also like to thank Axel Lindner, Alexey Petrov, Soeren Prell, Zack Sullivan, and Dieterich Wegener for useful discussions.

\section{REFERENCES}

1. K. Abe et al. [Belle Collaboration], Phys. Rev. Lett. 87, 091802 (2001).

2. B. Aubert et al. [BaBar Collaboration], Phys. Rev. Lett. 87, 091801 (2001); B. Aubert et al. [BaBar Collaboration], BaBar-PUB-01/03, SLAC-PUB-9060 [arXiv:hep-ex/0201020].

3. Y. Grossman, A.L. Kagan, Z. Ligeti, in preparation.

4. T. E. Coan et al. [CLEO Collaboration], Phys. Rev. Lett. 84, 5283 (2000) [ArXiv:hep-ex/9912057].

5. Y. Ushiroda [Belle Collaboration], in the Proceedings of the 4th Int. Conference on B Physics and CP Violation, February 2001, Ise-Shima, Japan, KEK Preprint 2001-13, KUNS-1717 [ArXiv:hepex/0104045].

6. B. Aubert et al. [BaBar Collaboration], BaBar-PUB-01/04, SLAC-PUB-8952 [ArXiv:hepex/0110065].

7. K. Osterberg, in the Proceedings of the Int. Europhysiscs Conference on High Energy Physics, Budapest, Hungary, July 2001.

8. S. W. Bosch and G. Buchalla, Nucl.Phys. B621, 459 (2002) [ArXiv:hep-ph/0106081]; Also see S. W. Bosch, in these proceedings [ArXiv:hep-ph/0109248].

9. M. Beneke, Th. Feldmann, D. Seidel, Nucl.Phys. B612, 25 (2001) [ArXiv:hep-ph/0106067].

10. M. Beneke, G. Buchalla, M. Neubert, C. T. Sachrajda, Phys. Rev. Lett. 83, 1914 (1999) [ArXiv:hepph/9905312]; Nucl. Phys. B591, 313 (2000) [ArXiv:hep-ph/0006124]; Nucl. Phys. B606, 245 (2001) [ArXiv:hep-ph/0104110].

11. A. L. Kagan and M. Neubert, CLNS-01/1756 [ArXiv:hep-ph/0110078]; Also see M. Neubert, in these proceedings.

12. T. E. Browder et al. [CLEO Collaboration], Phys. Rev. Lett. 81, 1786 (1998), [ArXiv:hepex/9804018].

13. C. Dallapiccola [BaBar Collaboration], invited talk at this symposium, see http://3w.hep.caltech.edu/HF9/program.html.

14. D. Atwood and A. Soni, Phys. Lett. B405, 150 (1997).

15. A. Datta, X. -G. He, S. Pakvasa, Phy. Lett. B419, 369 (1998).

16. A. L. Kagan and A. A. Petrov, [ArXiv:hep-ph/9707354]; Also see A. L. Kagan, in the Proceeedings of the 7th Int. Symposium on Heavy Flavor Physics, Santa-Barbara, CA, July 1997 [ArXiv:hep$\mathrm{ph} / 9806266]$

17. W. Hou and B. Tseng, Phys. Rev. Lett. 80, 434 (1998).

18. H. Albrecht et al. [ARGUS Collaboration] Z. Phys. C 58, 199 (1993).

19. A. Zimmermann, Diplom Thesis, University of Dortmund, April 1992.

20. Y. Chen and A. L. Kagan, in preparation.

21. T. Muta and M. -Z. Yang, Phys. Rev. D61, 054007 (2000), [ArXiv:hep-ph/9909484].

22. A. Ali and A. Ya. Parkhomenko, accepted in Phys. Rev. D [ArXiv:hep-ph/0012212].

23. For a review see Y. Nir, Lectures at XXVII SLAC Summer Institute on Particle Physics, IASSNSHEP-99-96 [arXiv:hep-ph/9911321].

24. Y. Grossman, A. L. Kagan, M. Neubert, JHEP 10, 029, 1999 [arXiv:hep-ph/9909297]. 
25. E. Berger et al., Phys. Rev. Lett. 86, 4231 (2001) [ArXiv:hep-ph/0012001]; M. Carena, S. Heinemeyer, C. E. M. Wagner, G. Weiglein, Phys. Rev. Lett. 86, 4463 (2001) [ArXiv:hep-ph/0008023]; Also see E. Berger, in these proceedings and references therein [ArXiv:hep-ph/0112062].

26. T. Becher, S. Braig, A. L. Kagan, M. Neubert, in the Proceedings of the Int. Europhysics Conference on High-Energy Physics, Budapest, Hungary, July 2001 [ArXiv:hep-ph/0112129].

27. L. Del Debbio, J. M. Flynn, L. Lellouch, J. Nieves, Phys. Lett. B416, 392 (1998) [ArXiv:heplat/9708008].

28. P. Ball, V. M. Braun, Y. Koike, K. Tanaka, Nucl. Phys. B529, 323 (1998) [ArXiv:hep-ph/9802299]; P. Ball and V. M. Braun, Phys. Rev. D58, 094016 (1998) [ArXiv:hep-ph/9805422].

29. G. Degrassi, P. Gambino, G. F. Giudice, JHEP 12, 009 (2000) [ArXiv:hep-ph/0009337]; M. Carena, D. Garcia, U. Nierste, C. E. Wagner, Phys. Lett. B499 141 (2001) [ArXiv:hep-ph/0010003]; D. A. Demir and K. A. Olive, Phys. Rev. D65, 034007 (2002).

30. M. Boz and N. K. Pak, [ArXiv:hep-ph/0201199].

31. J. H. Field, UGVA-DPNC 2001/1-185 [ArXiv:hep-ph/0101158]. 